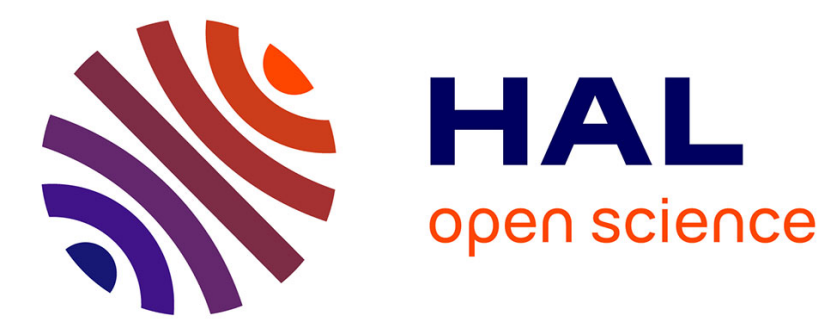

\title{
Les fosses funéraires de Cucuron (Vaucluse)
}

André Dumoulin

\section{To cite this version:}

André Dumoulin. Les fosses funéraires de Cucuron (Vaucluse). Gallia - Fouilles et monuments archéologiques en France métropolitaine, 1962, 20 (2), pp.323-333. 10.3406/galia.1962.2357 . hal01931234

\section{HAL Id: hal-01931234 \\ https://hal.science/hal-01931234}

Submitted on 25 Feb 2020

HAL is a multi-disciplinary open access archive for the deposit and dissemination of scientific research documents, whether they are published or not. The documents may come from teaching and research institutions in France or abroad, or from public or private research centers.
L'archive ouverte pluridisciplinaire HAL, est destinée au dépôt et à la diffusion de documents scientifiques de niveau recherche, publiés ou non, émanant des établissements d'enseignement et de recherche français ou étrangers, des laboratoires publics ou privés.

\section{(이) $\$$}

Distributed under a Creative Commons Attribution - NonCommercial - NoDerivatives $\mid 4.0$ 


\section{NOTES}

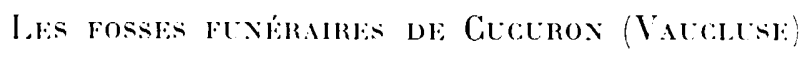

Au mois de fevrier 1959, a la suite du passage d'une rharrue défonceuse dans un terrain appartenant a M. Paulin Lanchier, silué quarlier des Conques a Cueuron. à $2 \mathrm{~km}$. jof environ aus.-F. de celle localité. M. Henri Reynier, dont les terres avoisinent ce lieu, remarqua des rharbons el des poleries brisées remontés par la charrue, glana quelques fragments divers ef recueillil mime un pelit vase intact ainsi que de nombreux rlous en fer. Il nous fit part de ses découverles. mais il élail trop lard pour sonerer a entreprendre des fouilles. le champ élant priel pour une plantation en melons. Ce n'est que l'année suivante. au débul de févier 1960. qu'une campagne de recherches permit la mise au jour de plusieurs fosses a incinéralion appartenant a deux époques différentes : les unes dalent du début du lle Jee du Fer ou la Tine I. soil le milieu du fer millénaire av. J.-C.. les autres, galloromaines. sont du I $^{\text {er }}$ siecle de notre ire (fig. 1).

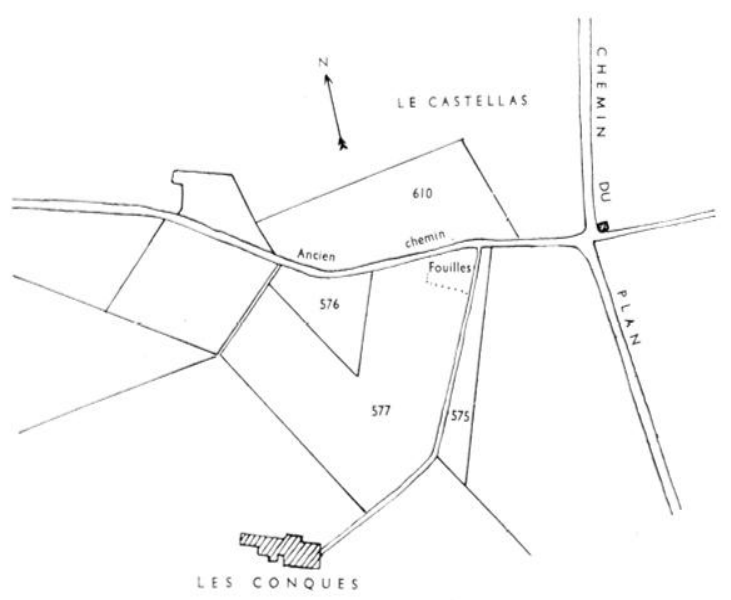

Figr. 1. - Plan Cadastral. Commme de cucurom Vaucluse, quartier des conques. Section E, dite du Plan.
Le lerrain dont il s'apit (parcelle $57 \pi$, seclion E dile du Plan est limité au Nord par l'ancien chemin d'Insouis dont on distingue encore le tracé malgré l'envahissement par les buissons el les arbustes. I'n mur vetuste en pierres siohes longe celte antique voie et sert de soulènement a un grand champ surélevé qui lui fait suile en direction du lieu-dit "Ise Castellas", où de nombreux vestiges préhistoriques onl été découverts. A l'Est. il est bordé par un petil chemin qui n'existe que depuis une trentaine d'années, aboulissant it la ferme des Conques ou habite M. Chanot. l'exploitant du terrain. C'est dans l'angle formé par ces chemins, au X.-L., que les fouilles ont été effectuées. Le sol remarquablement souple est constilué par un sable jaunatre, fin. provenant de la désagregalion des grès helvétiens sous-jacents qui forment la roche appelée "safre" dans le pays.

Nous avons pu ainsi comslater l'existence en ce lieu d'une premiere nécropole remontant a l'ápoque cello-liqure. puis d'une seronde de la période romaine. Mais les derniers dépòts onl souvent déranéé les précédents; les labours modernes onl. pour leur part. détruit partiellement de nombreuses tombes ou mélangé el dispersé leur contenu. Yéanmoins les fouilles entreprises ont foumi d'intéressants resultats.

Toutes les tombes. qu'olles soient préromaines ou romaines. se présentent sous la forme de fosses ovales creusées dans le sable à moins d'un motre de profondeur du sol actuel. Eilles sont pleines de terre noire el rontiennent des os humains calcinés. mélangés. au charbon provenant du bùcher, accompagnes: d'un mobilier funeraire fig. 2 . 


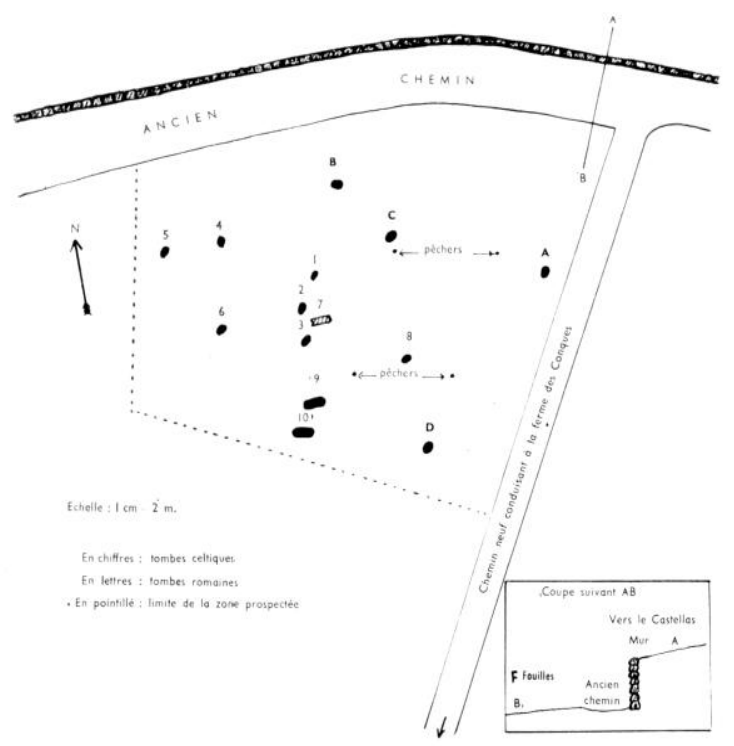

Fig. :2. .. - Cucuron. Quartier des Conques. Plan des fouilles avec emplacements des fosses funeraires.

\section{Sépullures pré-romaines}

Tombe 1. Fosse bouleversée, at mohilier éparpillé et incomplet. Ont été recueillis :

10 un bracelet fracmente. en bronze. lige lisse fermee. de section quadrangulaire (I) : 5 ('m. 5): h. : 0 cm. 45: cepais. de la tige: 0) (am. 2$)($ fig. 3 a):
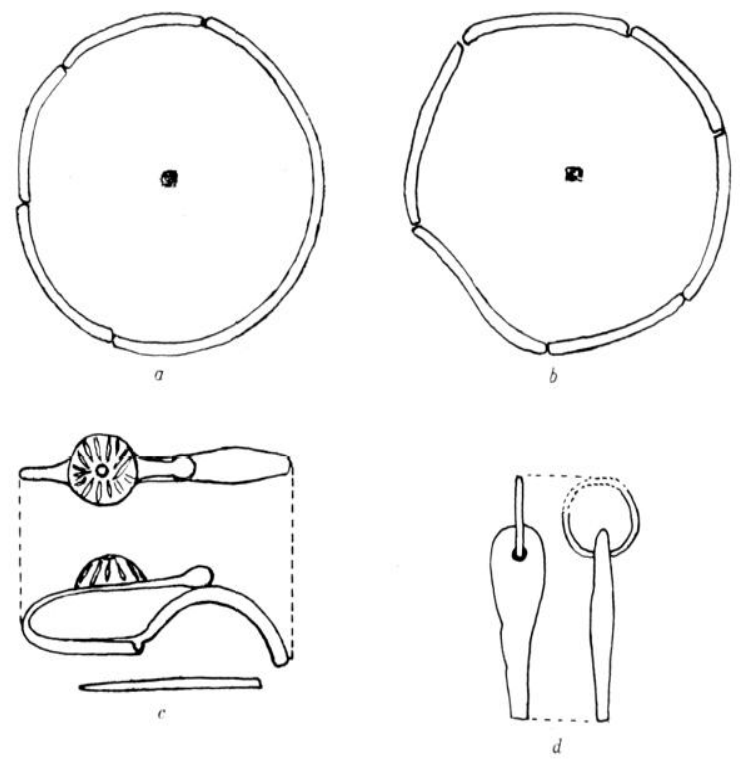

Fis. 3. - Bracelets, fibule et pendeloque en bronze de la tombe $A$. 2o un aulre bracelel deformé, brisé en plusieurs morceaux. du mime genre que le preceident (fig. 3 b):

$3^{0}$ une fibule en bronze donl il manque le ressort. L'are, un peu aplati, se termine par un porle-agrafe que prolonge une lige coudée renllée a son extrémité qui vient s'appuyer au dos de l'are. Celle tige est claroie en disque. a la partie supérieure duquel se l rouve rivé une bosselte en os a décor incisé rayonnant. C'est une forme caractéristique de la Téne $1^{1}$ (longueur : $4 \mathrm{~cm} .4$; haul. : $1 \mathrm{~cm} .5)$ (lig. $3 \mathrm{cl}$ :

to une pendeloque constiluée par une lige de bronze plate avec perforation terminale dans laquelle passe un pelil anneau filiforme mobile (longueur : $3 \mathrm{~cm}$. 2) diamelre de l'anneau : $1 \mathrm{~cm} .4)(\mathrm{fig} .3 \mathrm{~d}$.

Trmbe B. Cuetle fosse de 0 m. Si) sur () m. 65). profonde de 0 m. 20. Irouvee en place ¿ $0 \mathrm{~m} .60$ du niveau du sol. avail la parlicularité d'être délimitée intérieurement par un cordon de petits blocs irréguliers de wros helvétien (fig. 4 et s)). Elle contenait :

10 un petit gobelet rylindro-conique, court. non lourné en argile grise criblée de grains

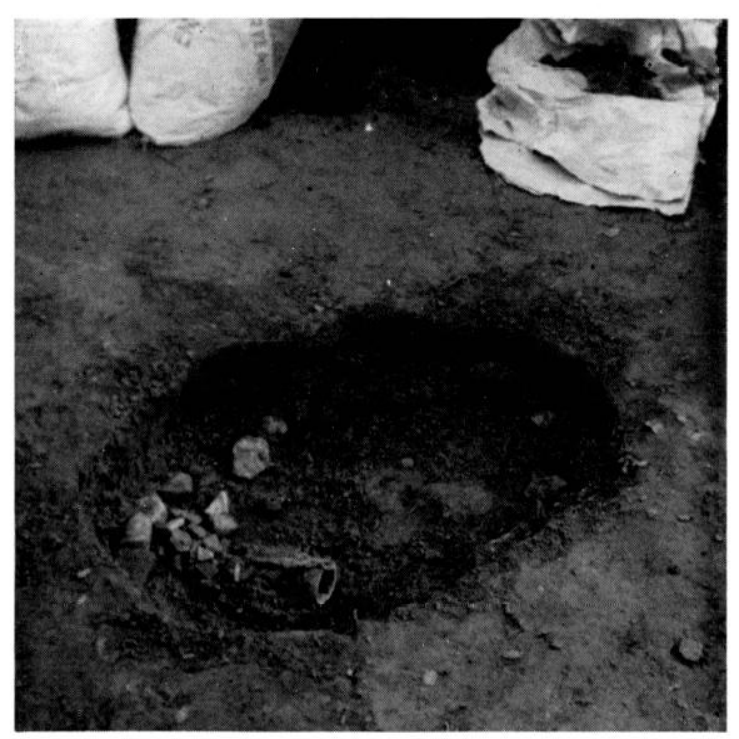

Fig. 4. - - Tombe $B$ en fin de fouille.

(1) Cne fibule d'un type tries proche de notre exemplaire a ete trouve a levey Suisse fe chaton

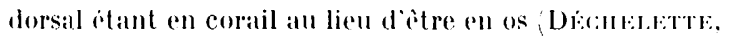
Mantel, II, p. 1218, fị. 5.33, n" $15 \%$. 


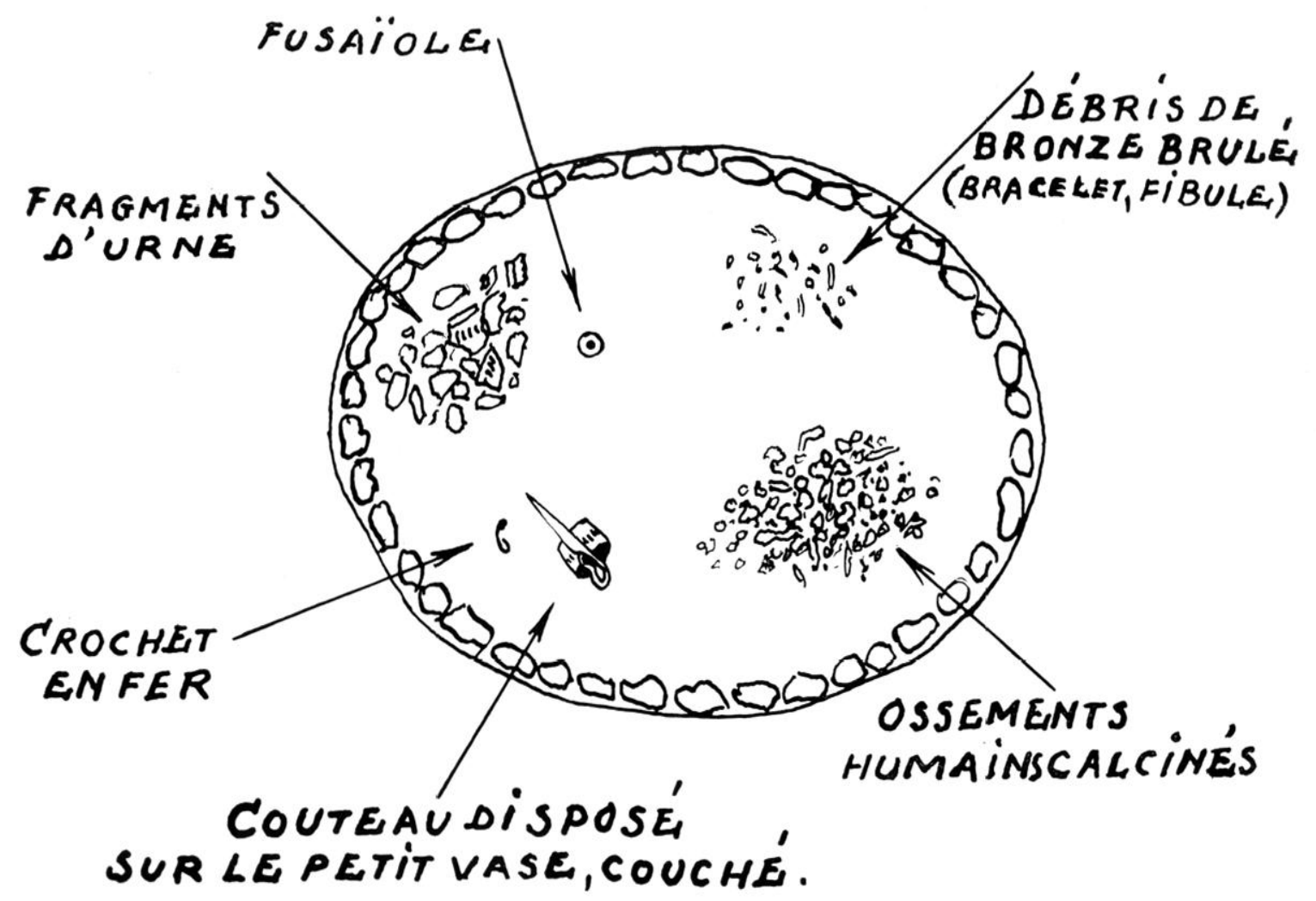

Fig. 5. ... Plan de la tombe B.

de calcaire. i fond plat el parois épaisses orne au liers supérieur d'une rangée d'incisions obliques (H. : 4 cm. 5) ; I)O : 7 (m. 2; I)B : (j centimetres : epaisseur de la paroi : 0 cm. 6 (fig. 6 el $7:$;

zo plusieurs fragments d'une urne a col arqué avec encoches obliques allongees. fond plat (fig. 8 :

$3^{\circ}$ une fusaïole bi-Ironconique en lerre grise modelée grossierement (II. : 2 cm. i): I) : 4 centimelres: (fig. 9 c: :

$4^{0}$ un couleau en fer a lame plate dont la soie. repliée sur elle-meme dans le plan de la lame, vient se raccorder par une volute a la base de la poignée (I. : 15) (cm. 4: larg. : 2 centimitres: long. de la poignée : 5 centimetres). Ce couteau se (rouvait placé horizontalement sur le pelit vase, décrit en premier

2: Ce couteau a soit de forme singuliere rappelle ceux du mont Beuvaly et de Stradonity La Tene II dont l'extremite amincie se recourbe en crochet annulaire (DischlolitTl, p. 1363, fiy. 5) 59, $n^{n s}: 2$ et 3 ef p. 1366 .

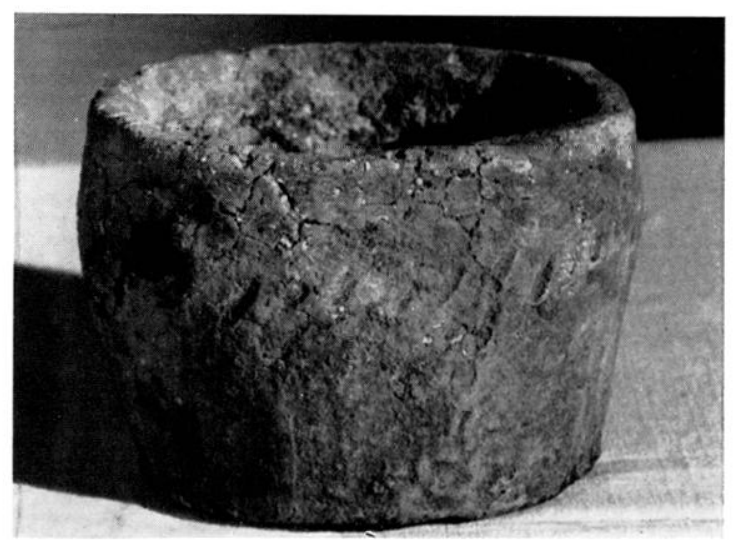

Fig. 6. - Gobelet orne de stries obliques do lit tombe B isr. nat.

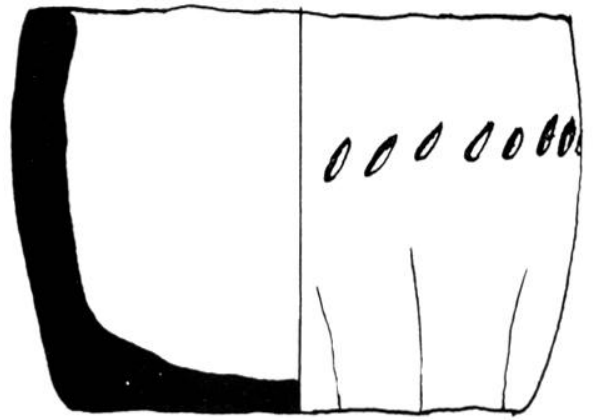

Fig. 7. - Coupe du gobelet de la tombe B. 


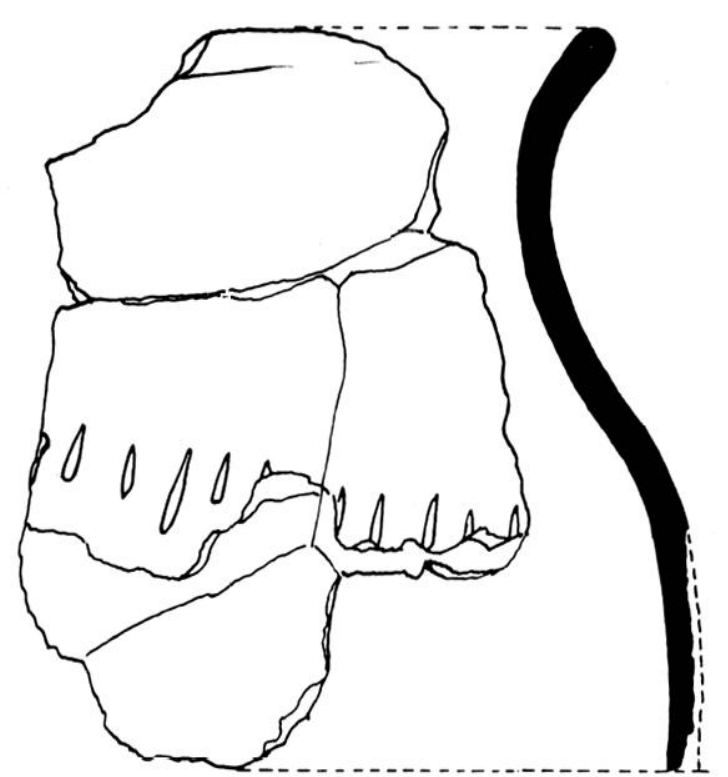

Fig. 8. - Fragments de l'urne de la tombe B.
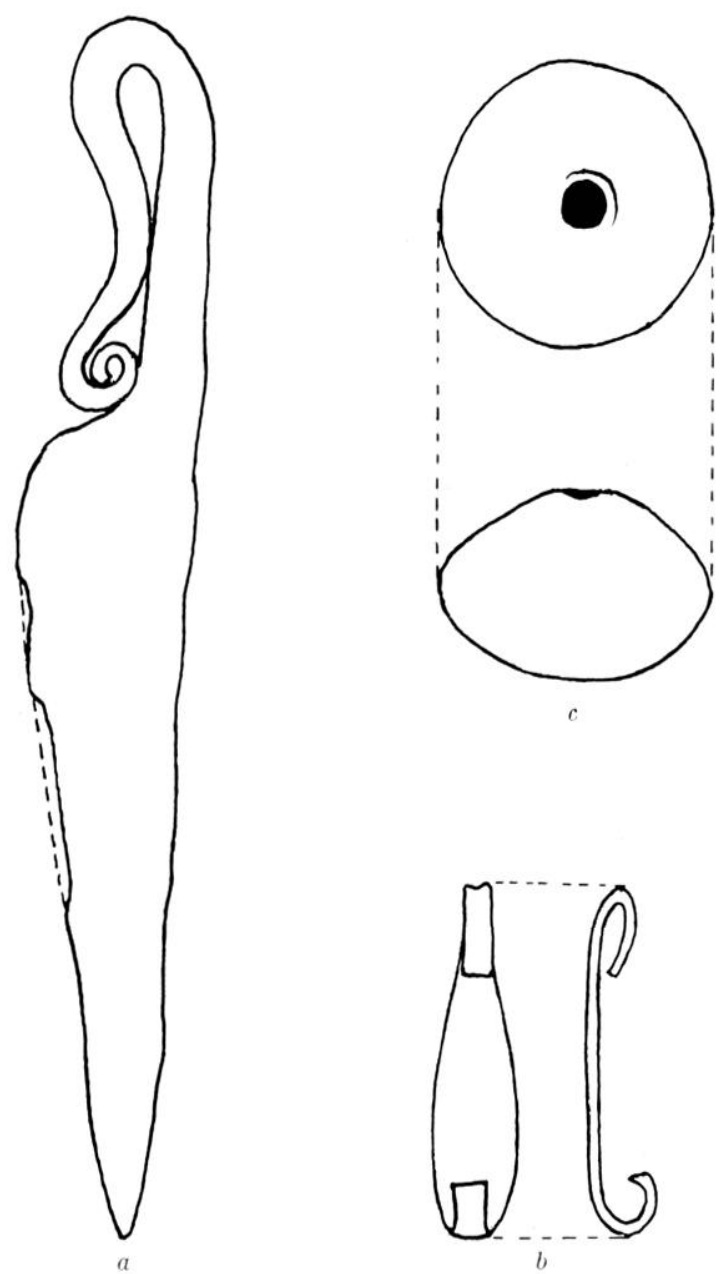

Figr. 9. - routeau en fer a soie repliece en volute (a) erochet en fer (b) et fusaīole (c) de la tombe B. lieu. qui étail rouché sur le còté (cf. photo) (fig. 9 a) ;

5o une plaquelle ovale allongée en fer. à extrémités terminées en forme de crochet (I. : 4 cm. 5) ; larg. max. : 1 cm. 3), qui est probablement une agrafe de ceinture (fig. $9 \mathrm{~b}$ ) ;

(;o une cinquantaine de débris de bronze fondus et déformés parmi lesquels on peut reconnaître les fragments d'un bracelet et d'une fibule dont il n'est pas possible de préciser la forme.

Tombe $C . \quad$ Fosse ovale, en place. a $0 \mathrm{~m}$. 70 de profondeur $(0 \mathrm{~m}$. 65) de long et $0 \mathrm{~m}$. 45) de large). Elle contenait :

$1^{\circ}$ une grande fibule en fer à are épais arrondi au centre, aminci a chaque bout. terminé d'un còté par un porte-agrafe asse\% court prolongé par un appendice a trois petits renllements bulbeux. qui se rabat sur l'arc sans le loucher. L'autre extrémité est recourbée en forme de spire. enserrant une lige de fer de 2 cenlimilres de long autour de laquelle sont enroulés deux éléments de ressort en bronze (long. : 9 centimetres; h. : $2 \mathrm{~cm} .5)^{3}$ (fip. 10) ;
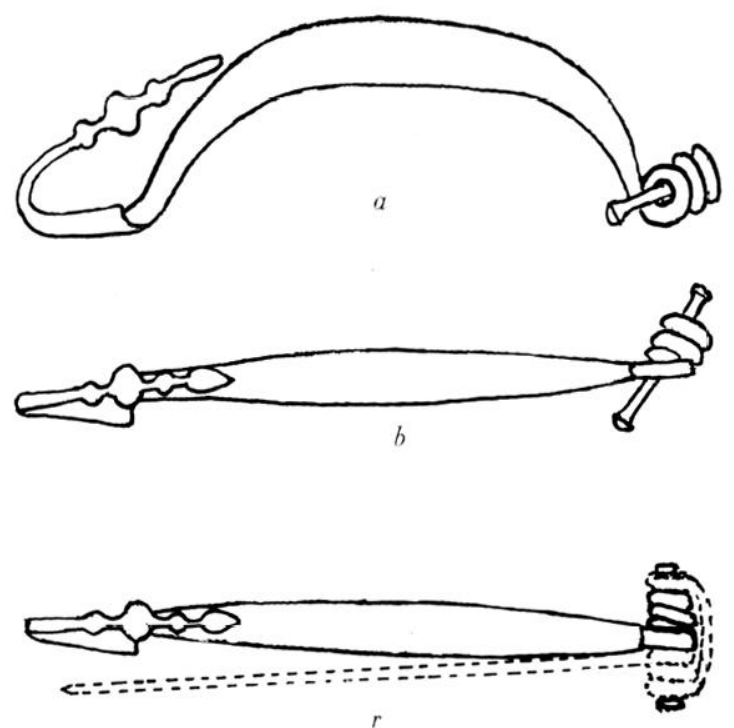

Fin. 10. - Fibule en fer de la lombe l: (a b) el reconstiture (r).

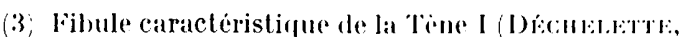
p. 1:18, fig. 533, $n^{\circ} 11$ a 17 , representé dans le niveau 111 du Cayla de Mailhac début ve-fin nes. av. J.-C.. O. et J. Tafraxes, le Cayla de Mailhac (Aude), (arcassonne, 193x, p. 33, fig. 7 et $x$. 
20 fragments d'un vase non lourné du lype urne. ell areque a degraissant ralcaire. dont une erande partie a pur elre reconstiluée. Fond plal, panse peigné arece rangée doincisions courtes horizonlales placés assez haul a la base du col; bord a livere déversée (II. : $2: 3$ (cm. 8 ; 1). du fond: 10 rentimbles) (fiw. 11 ;

30 plusieurs fragments d'un vase du mème genre que le prédent. mais de dimensions moindres.

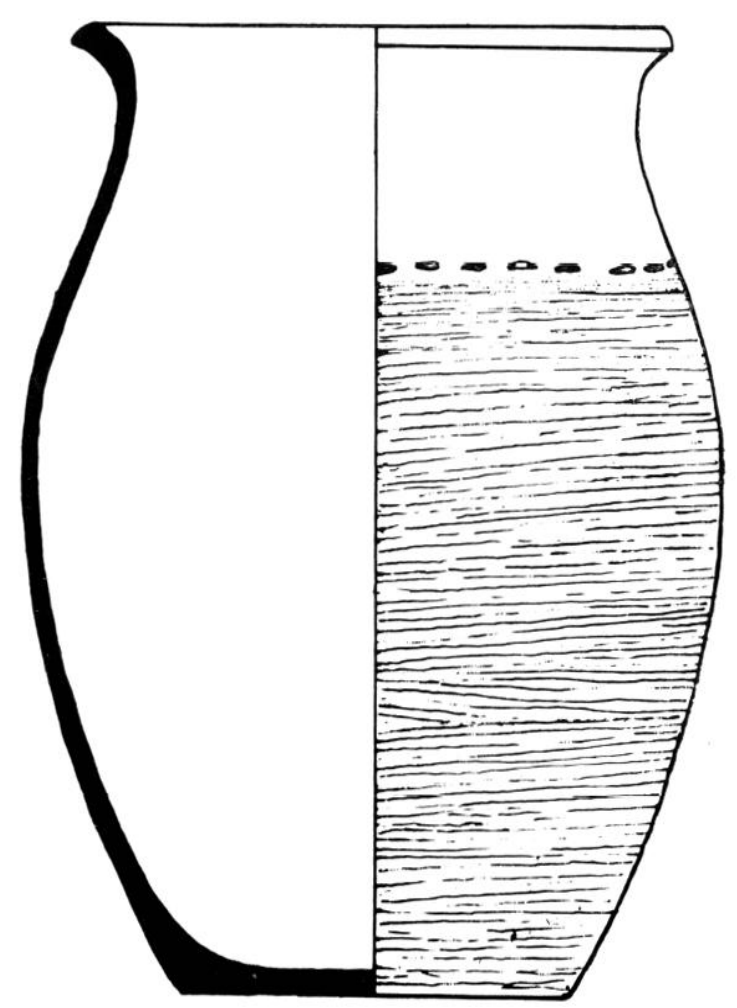

Fig. 11.-- Irne cincraire de la tombe C (reconstitution .

Tombe D. - Narquée par quelques osisements brùlés, en pleine lerre, accompagnant une pointe de lance en fer, lres délériorée. Celle arme. de forme allongée, triangulaire. a nervure médiane apparente el douille pour l'emmanchement. mesure 18 cm. jo de long et 3 centimetres de large (fig. 12).

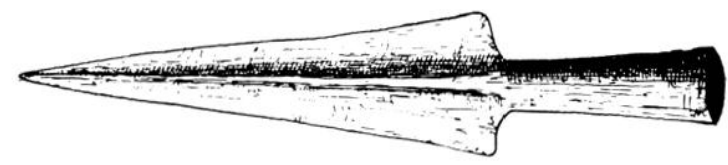

Fig. 12. Pointe de lance en fer de la tombe D.
Sépullures rmmaines:

Tomber 1. a) Éruelle an lerre rouger

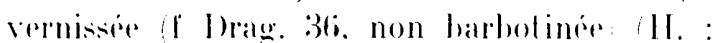

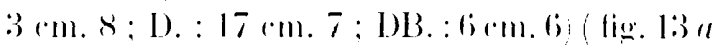
al (lig. I) e):

b) bouteille a panse prismalique de section quadrangulaire en verre lransparent bleu rair. goulot etroil of anse coudere forme 1.1 de Jorin-Jean sous le fond. ornement tribobe dans un reerele. dont le relief. allabbli par lesure. indigue que ere tlacen avail ble ulilisé avant d'ide depose dans la lombe (II. :

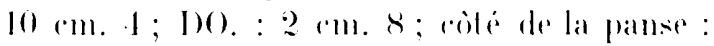

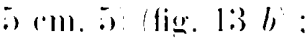

c) moilie gauche de lamper is huile "n lerte jaunale a couverle marron, here incomplest. a volutes laterales:

d) autre fragment de lampe. en lere errise, du meme type. Ires déléroré par le feu.

Tombe 2. a) Exuelle an lerre rouge vernissee (f. Drag. 36 a rebord barbotiné sinistrogye (H. : :3 cm. 8: 110. : 16 cm. 3; DB : 6 cm. T) (fig. 1:) c et fig. lis f);

b) pelile potiche ovoüde de La Graufesenque en lerre fine rouge vernisiere male (forme Hermet 8 ) avec moulure en haul de la

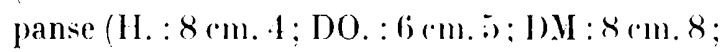
DI3 : 3 cm. 9) (fig. 13 $d$ el fix. 15 c) ;

c) écuelle en lerre fine grisalre lendre a couverte marron clair (imilation un peu réduile de la forme l)rag. :36) (II. : 3 (cm. 6; DO. : 14 centimetres; I)B. : ci rentimetres) ;

d) fragments d'une urnetfe en terre commune (D). de base : $4(\mathrm{~cm} .5)$ :

e) fragments d'une autre petile urne (bord et panse);

f) lampe de belle facture en lerre grise dure. a engrobe marron clair; l'exlrémilé du bec. a grandes rolutes, manque. Le dessus est orné d'une tête juvénile de bacchanle. de face, a fort relief (II. : $2 \mathrm{~cm} .7$; L. : 8 cm. 1; 1.: 7 ('m. 6); sous le réservoir. signe de série en creux (fig. $14 a)$;

g) vase a liquide à anse en lerre jaume friable. a panse ronde. fres lragmente. 

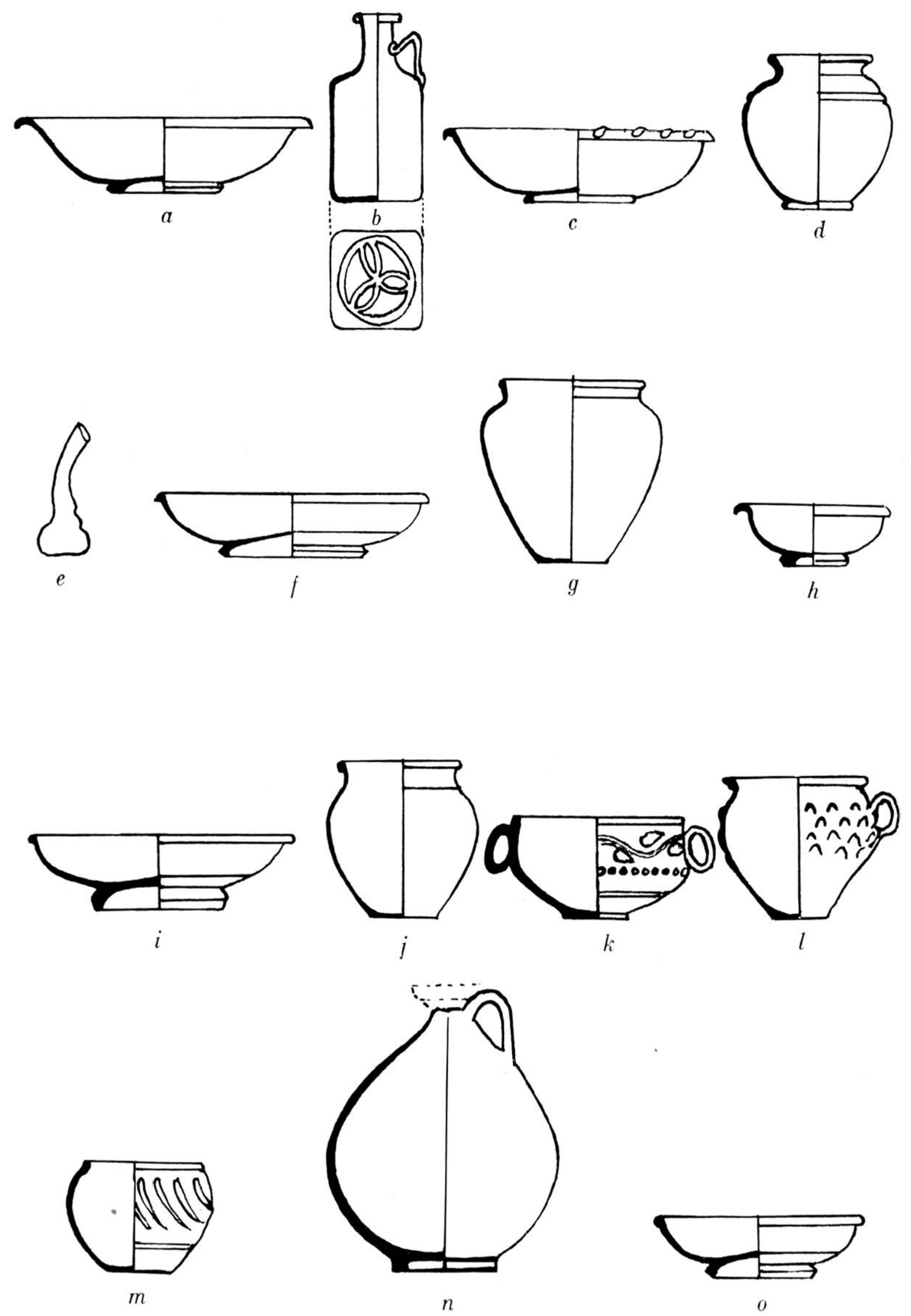

Fig. 13. - Vases des tombes $1(a-b), 2(c-d), 3(e-f), 4(i-j-k-l), 5(g-h), 6(m), 7(n)$ el $8(0)$. 

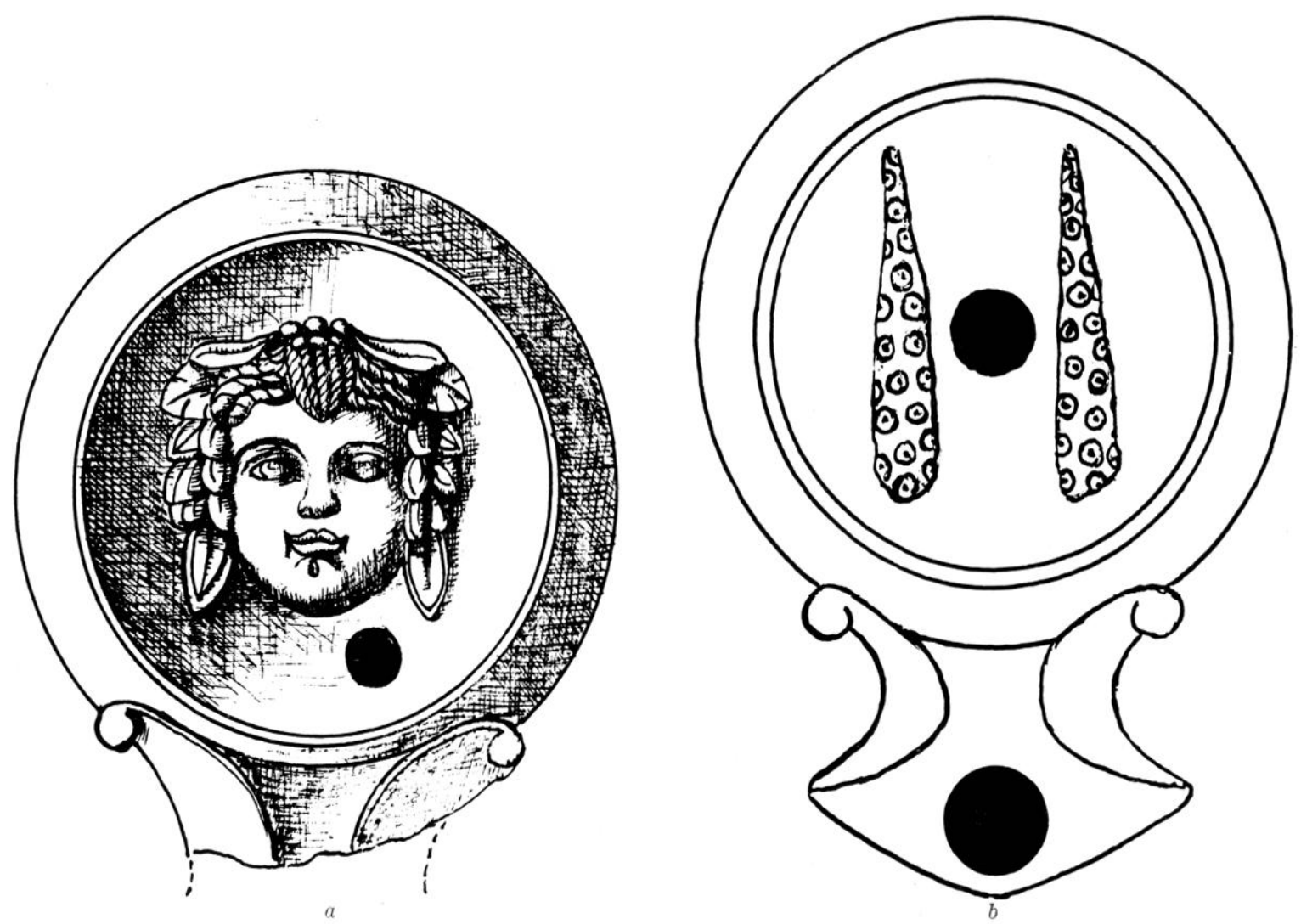

Fig. 14. - lampes des tombes 2 (a) et 1 (b).

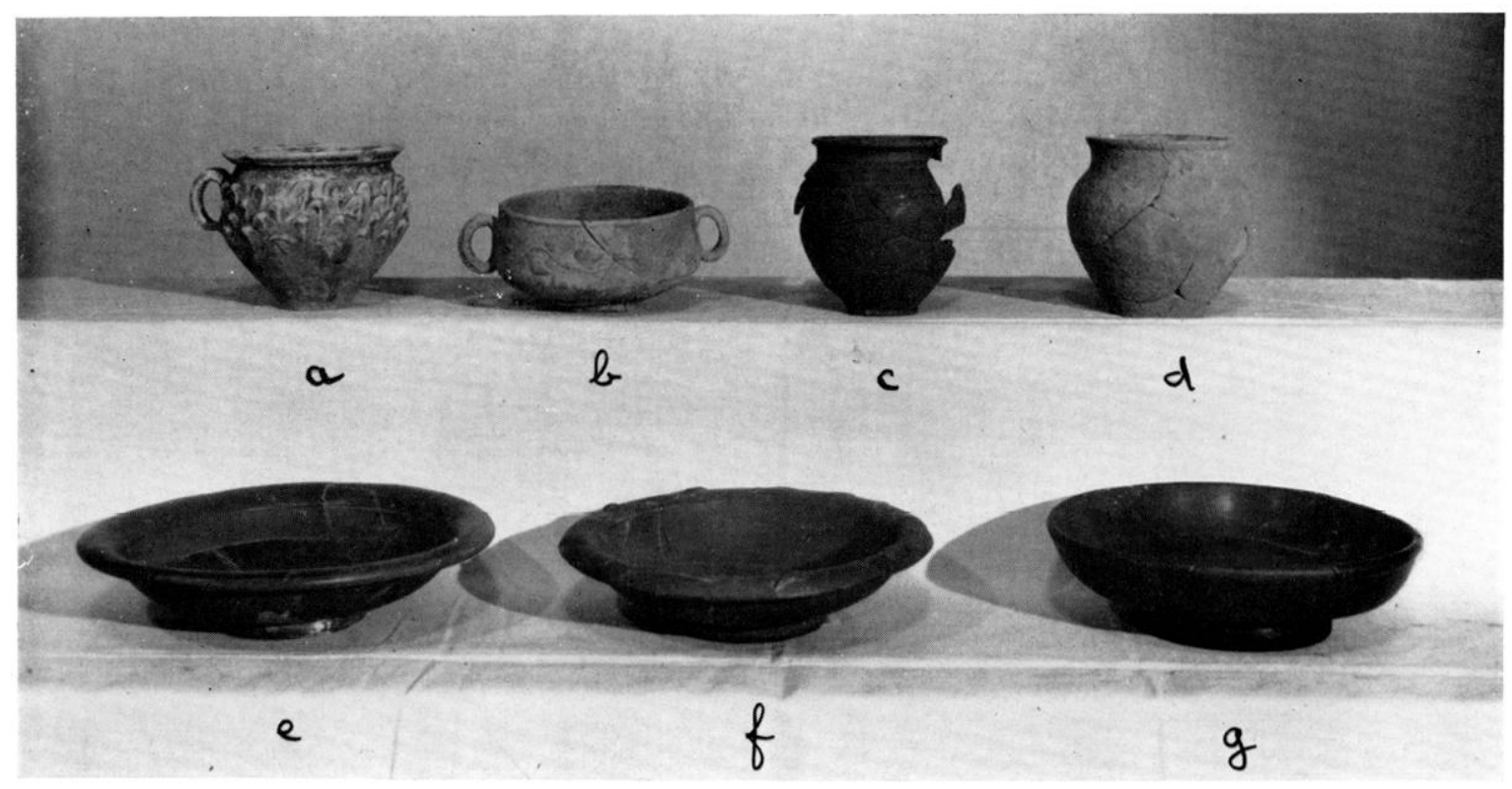

ligy. 15. Viases des tombes 1 (e), 2 (c-f), $3(a), 4(a-b-d)$ 
Tombe 3. (1) Écuelle an lerre rouge vernisiere (f. Jata. 18 estampillé intérieurement : OF. VIRILI (atelier de lirilis

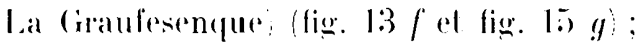

b) "lacrvmaloire" en verre bleu clair. aplati el deformé par le leu (II. : 10 cm. 7 : épais. :

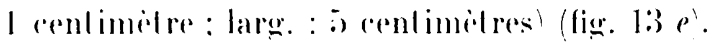

Tomber t. a) Éruelle an lerre rouger vernissere avere marque de potier assez fruste:

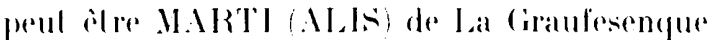

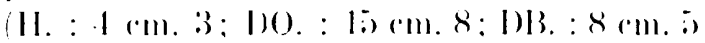
(1i... 1:3 $i:$

b) turnelle en ferre erise. rommune, a fond

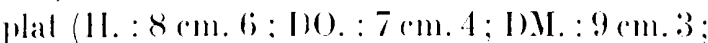

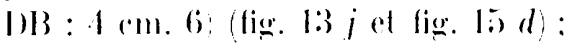

c) Lasse en aroile grise fine. a deux anses. fond plal. forme rarenée. ornée sous le bord d'un rineau de leuilles d'eau. i la barbotinet

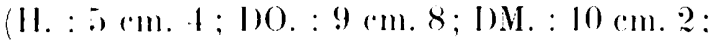
IIB. : A centimioless (tig. 13 li al fig. 15) b) :

d) pelil vase a parois lines. à une anse. panse surhausiée piquelée de prolubéranees arcileuses en forme d'écailles de pomme de pin dans sa moilie supérieure (lerre jaunatre i engobe brun clair). Fond plal elroil (H. :

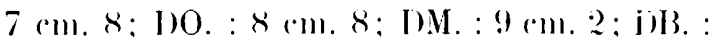

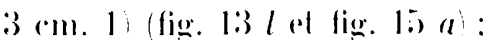

e) lampe complede a bec oblus el volules. ornée sur le dessus de deux massues a nodosiles apparentes, disposees symetriquement de chaque colé du trou d'alimentalion (terre grise à engobe plus foncé) $)^{5}$ (11.: 2 cm. i): L. : 9 (entimilres: 1 . : $6 \mathrm{~cm} .2)($ fig. $14 b)$ :

f) dix clous en fer forgé a labe plate plus ou moins ronde. el lige i section quadrangulaire. la plupart tordus.

(1) Vase semblable fig̣uri dans : R. Borlan, Réenles decourerles archeologiques antr Blaiss (lar), dans : Cahiers ligures de prehisloire el darcheologie, 8, 1959, p. 9.1, fig. 6, no 2. lipoque flavienne.

(5) Motif ornemental connu sur une lampe de même Iype provenant de Vaison, al Muse Calvet (P. Dl: BRI:

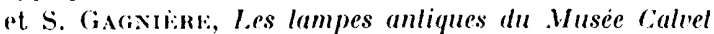
d'Avignon, Carpentras, 1937, p. 16, $n^{\circ} 197$; et sur un fragment trouve a Glanum (P. 1)t BRt'x, Lampes anliques en argile du musec des Alpilles a Saint-Rémy-de-

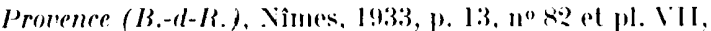
1105 .
Tombe is. a) Petil bol en lerere rouge vernissere a rebord lisse (f. I)rag. 35) (II. :

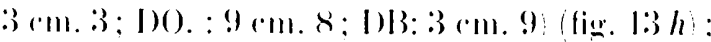

b) turnelle en lere commune. de lype rourant (II. : 10 rentimbles: I)0. : 9 am .2:

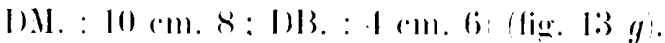

T'umbe (i. a) Trois ionelless semblables en ferer vernissere rouge ou noire anx endroils

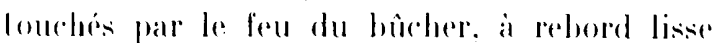

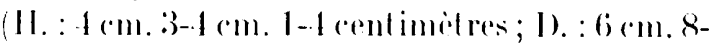

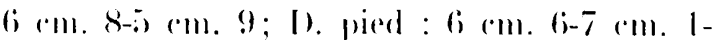
(i r.III. 2):

b) fragments d'une urnelle tres moreelée:

c) fragments d'un vase a liquide en terre jaune. de forme ordinaire:

d) lampe incomplibe en lerre grise. lype a volutes ef bec rond, ormement tries llou sur la cuvelle : quadrupede courant à qauche:

p) petit bol rond a fond plat. en polerie line marron. a panse orne sur les trois quarts supérieurs d'une rangée de balons obliquess barbolines 6 (11. : 6 ( $\mathrm{ml}$. 2: D) : : 7 (.m. :3: I)B. : :3 cm. ()) (fig. 1:3 m):

f) goulot d'un "lacrymaloire "déforme par le feu ;

g) une vinglaine de clous en fer.

Tombe \%. Tuile rourre-joint. Iongue de (ii) (om. 5) large de 21 el 17 centimetres. paraissant avoir recouvert en parlie une fosse. acrompannee d'un fond d'amphore a pied et d'un pelit vase a liquide casse en deux morreaus: le goulol manque. la panse es piriforme (II. : $14 \mathrm{~cm} .8$; I)M. : 14 ('m. :3: 1)(o.: $2(\mathrm{~m} .6 ; \mathrm{I}) \mathrm{B} .: 6(\mathrm{~m} .8)(\mathrm{fig} .13 n)$

Tombe \&. Fosse ovale en place. a 10 m. 万o) de profondeur. longue de $0 \mathrm{~m}$. To. large de 0) 111 . ti) el haule de 0 m. 25. Elle conlenail:

a) une petite écuelle en polerie fine a vernis jaune marbré de rouge (f. Drag. 18) alelier de

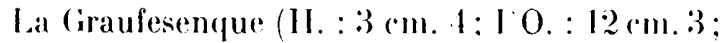
I)l3. : 6 cm. 4) (fig. 1:) o!:

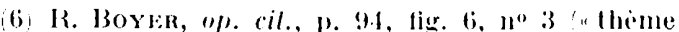
typique de la période $60-80$ ". 

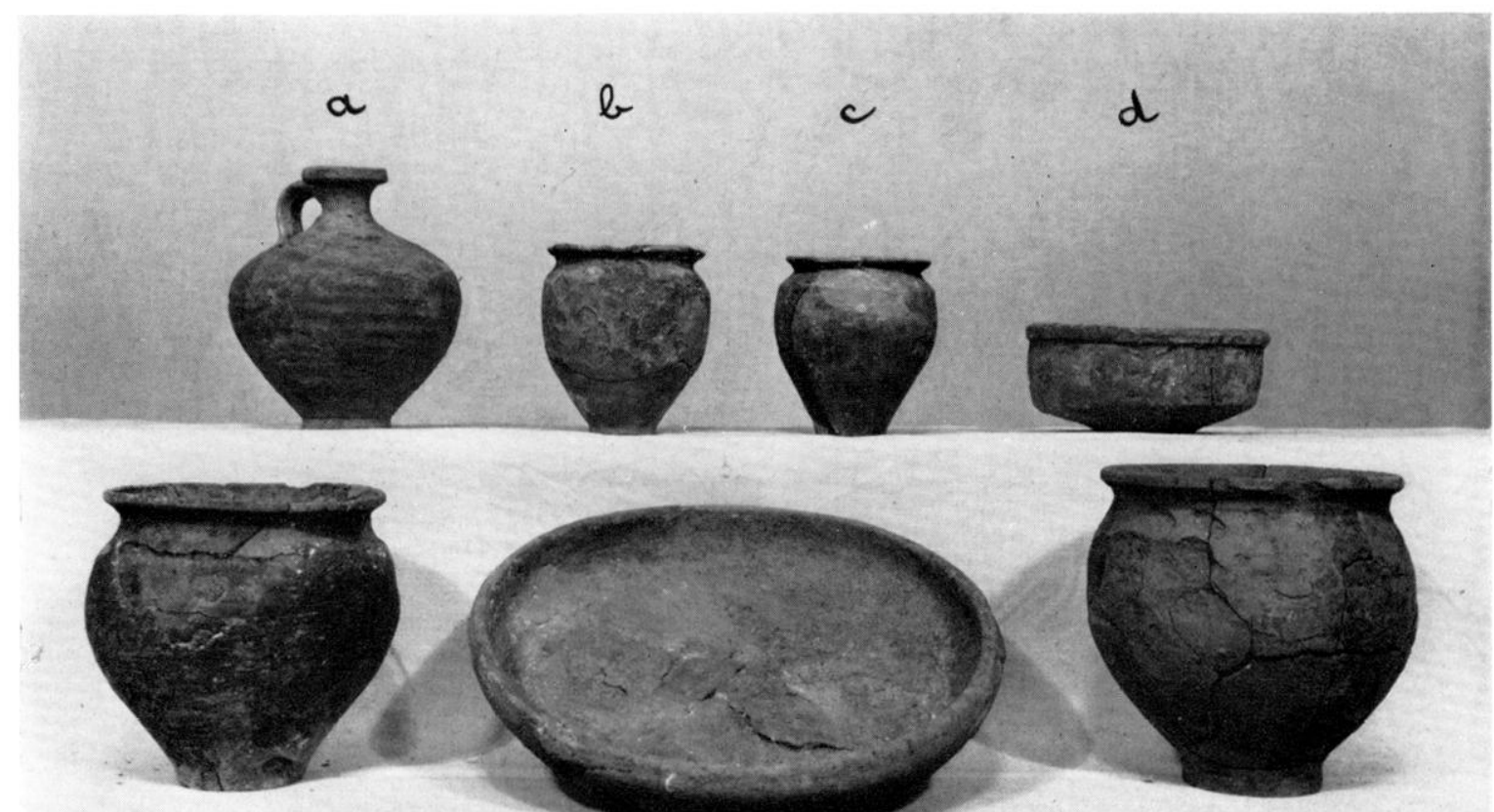

e

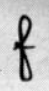

g

Fin. 16. Vinses de la tombe 9.
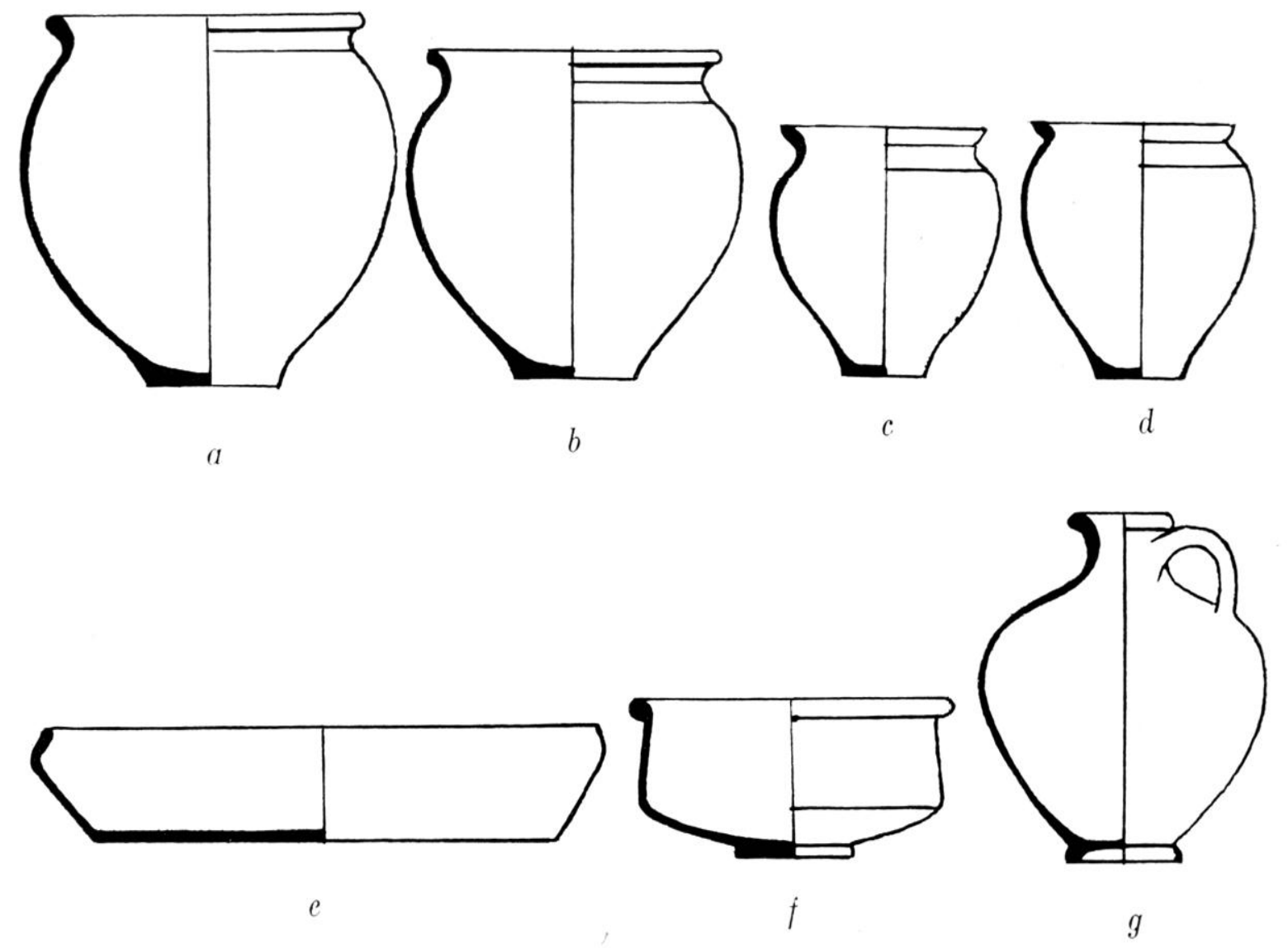

F1. 17. - Vases de la tombe 4. 
b) un moyen bronze de CLAUDE Ier (41-54 ap. J.-C. ; Cohen, 84) ;

c) un fragment de col de "lacrymatoire" brûlé ;

d) quatorze clous en fer.

Tombe 9. -.. Grande fosse de $1 \mathrm{~m} .90 \times$ $1 \mathrm{~m} .30 \times 0 \mathrm{~m}$. 35, qui contenait sept vases en poterie commune et une sorte de terrine ovale en argile jaunattre friable, en multiples fragments (fig. 16 et 17) :

a) écuelle plate à bords obliques et livre rentrante (H. : $4 \mathrm{~cm} .2$; DM. : $24 \mathrm{~cm} .5$ DB. : 20 centimètres) (fig. $16 f$ et fig. $17 \mathrm{e}$ );

b) bol caréné surbaissé à fond plat étroit (H. : 5 cm. 8; DO. : $13 \mathrm{~cm} .5 ;$ DB. : $4 \mathrm{~cm} .7$ ) (fig. $16 d$ et fig. $17 f$ );

c) urne en terre rougeâtre (devenue grise par la chaleur) à panse ronde et pied étroit (H. : $14 \mathrm{~cm} .3$; DO. : $13 \mathrm{~cm} .4$; I)M. : $15 \mathrm{~cm} .1$; db. : 6 centimitres) (fig. $16 \mathrm{~g}$ et fig. $17 a$ );

d) urne de mème type mais de taille un peu plus faible (II. : $12 \mathrm{~cm} .8$; DO. : $12 \mathrm{~cm} .2$; I)M. : 14 centimelres ; I)B. : $5 \mathrm{~cm} .4$ ) (fig. 16 e et fig. 17 b);

e) urnelle a col oblique, panse surhaussée a léger épaulement (H. : 9 cm. 4 ; DO. : $8 \mathrm{~cm} .4$; IDM $: 9 \mathrm{~cm} .2 ;$ I)M. : $3 \mathrm{~cm}$. 5) (fig. $16 b$ et fix. 17 c) ;

f) urnetle semblable à la précédente, plus effilée vers le bas (H. : $9 \mathrm{~cm} .6 ; 1) 0 .: 8 \mathrm{~cm} .5$; I) M. : $9 \mathrm{~cm} .3 ; 1)$ B. $: 3 \mathrm{~cm} .6$ ) (fig. $16 \mathrm{cet}$ fig. 17 d);

g) vase a liquide à double sillon circulaire au col, anse arrondie (II. : $13 \mathrm{~cm} .2$; I)O.: $4 \mathrm{~cm} .2$; D). : $11 \mathrm{~cm} .8$; D B. : $4 \mathrm{~cm} .6$ ) (fig. 16 a et figr. 17 !) ;

h) terrine ovale dont fout be bord mancque (II. actuelle : 16 am. 6 ; grand diamelre : 23 cm. 3; petit diametre: 16 cm. 5).

Tombe 10. -. Fosse allongée aree une amphore placée sens dessus dessous (lype a goulot court et deux anses arrondies engainanles, llanes bombés). Le pied manque. (II. actuelle : $4 t$ centimetres; DO. : $11 \mathrm{~cm}$.): I). panse : $42 \mathrm{~cm}$. 5) (fig. 18). A proximilé se trouvaient les fragments des récipients suirant: :

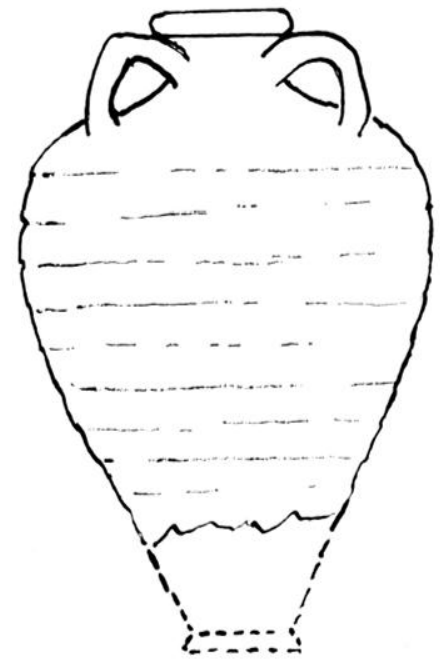

Fig. 18. - Amphore de la tombe 10.

a) écuelle basse, de même forme que celle de la tombe $9(\mathrm{a})$, ayant subi fortement l'action du feu (II. : $4 \mathrm{~cm} . ; 7$ D)O. : 25) cm. 5); DB. : 20 centimilures);

b) urnetle en trìs mauvais état et incomplete (I)B. : $3 \mathrm{~cm}$. 5).

Signalons enfin la trouvaille, dans les déblais, de deux hachettes brisées en pierre verte polie et de deux fragments de lames en silex dont une est a encoche avec fines. retouches.

Voici donc un lieu où, à deux époques différentes mais non successives. des Celto-Ligures. puis des Gaulois de l'époque romaine ont enterré leurs morts en pratiquant le mème rite : l'incinération avec dépòt funéraire. d'une façon dont la ressemblance n'est pas sans surprendre. Les tombes gallo-romaines semblables ne sonl pas rares dans la régionn elles remontent généralement a la fin du

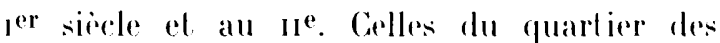
Conques apparliennent a la ze moilir du

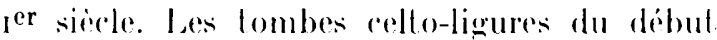
de la Tene sont au contraire peu communes. On sail qu'a celle ćpoque les cimetiores bilaient

(7) A Sannes, an quartier des clots distant denviron 5 kilomitres a l'list de l'endeoit qui nous occupe, nous avons foulle en l951 un ensemble de :2 stepultures a incineration des ger et re siecles ap. .J.- : : Rerherches archeologiques dans la région dilpl (tameluse), dans

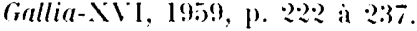


rapprochés des oppidums. "au dehors des remparts el sur les pentes de la colline supporlant l'habitat ${ }^{8}$." Or celui-ci est lout proche : a quelques centaines de mètres au Nord se trouve une éminence boisée qui porte le nom de : "Castellas ". Nous l'avons mentionné au début de cette étude. Ses abords ont donné des armes et des outils en silex taillé ainsi que des haches en pierre polie associées à de la poterie grossiere (collection Henri Reynier, a Cucuron). Au sommet, on distingue des vestiges d'antiques murailles dans les buissons. Il esl permis de penser que ce sile, après avoir été ulilisé par des hommes néolithiques,

(8) II. de Girm-licand, Préhistoire el Prolohisloire, Marseille, 1931, p. 15. a servi d'oppidum à des éléments de la tribu des Dexsiviates qui s'étaient établis sur la rive droite de la basse Durance, au pied méridional du Luberon. dont le camp principal. le Castellaras de Cadenet. n'est d'ailleurs qu'à environ 4 kilomètres, au sud-Ouest. Quant aux tombes romaines, elles impliquent un habital qui n'était plus le Castellas, mais un locus comme à Sannes, dont l'emplacement peut ètre situé à 300 mètres environ vers le Sud, dans un champ près d'un point d'eau, où les labours mettent souvent au jour les vestiges caractérisant des constructions : tuiles diverses, moellons, enduits muraux et fragments de béton, lessons d'amphores et de doliums, elc.

André I)truolims.

Le castelicy (iabale du Roc de la Fare (Commune de Laval-id-Tarn, Lozìne)

Le Roc de la Fare est le nom donné à un entablement calcaire, limité sur la moitié de son périmetre par des escarpements naturels, qui domine le hameau de Montredon, sur le Causse de Sauvelerre (commune de Laval-duTarn, Lozère), à 7 kilometres à vol d'oiseau au

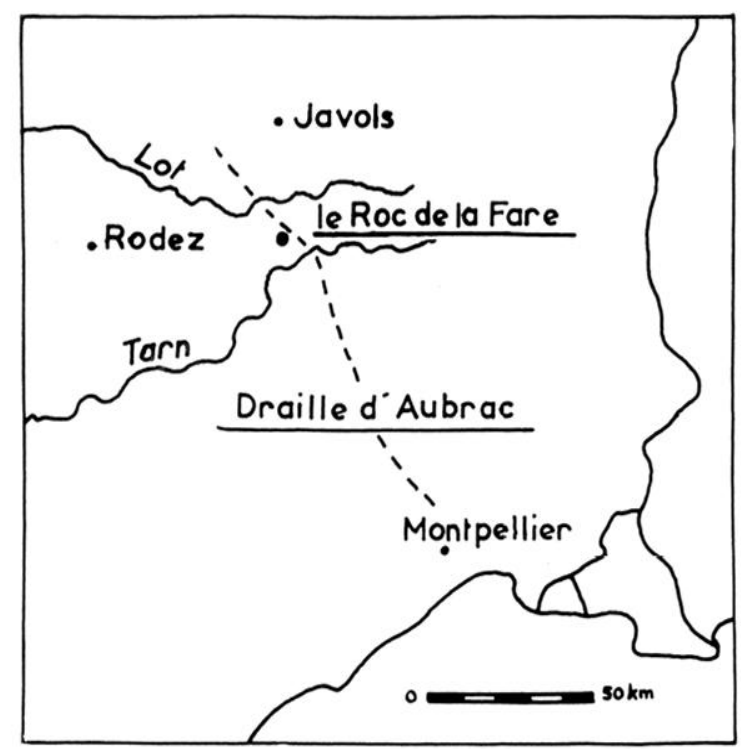

Fig. 1. - Situation du Roc de la Fare.

1) Nom local : lou Rom de la Farro (cadastre du xixe siecle, section $13, n^{\circ} 17 \pi$, la Farre! l.e premier
Nord-Ouest de Sainte-Énimie et à 1 kilomètre de la Draille d'Aubrac (fig. 1 et 2 ). Ce promontoire présente une surface a peu pris plane, constituée par un lapiaz à strates sensiblement horizontales, de forme presque rectangulaire : $80 \times 130$ metres (fig. 3). Sur les còtés Est. Nord et Nord-Ouest. les pentes sont couverles de grands entassements de pierres. vestiges de murailles en partie écroulées. tandis que la table calcaire du sommet est, constellée par endroits de pelits lessons de poterie $^{2}$. Il y a la visiblement une de ces forlifications prolohistoriques du lype éperonbarré. comme on en rencontre en assez grand nombre sur les Crands Causses ${ }^{3}$. L'intéròl du site réside dans le fail que, contrairement a la plupart des cas jusqu'à présent observés. des débris de céramique apparaissent à la surface du sol.

terme correspond à lancien provengal ranc: rocher : quant au second, il est le continuateur du germanique fara (cf. Vixcext, Toponymie de la France, Bruxelles, $1937, \leq 362$.

(2) Ces restigres ont ete remarques et signales pour la premiere fois par Mlle de Mariac: (Bullelin de la Sociele Prehishrique Française, 1958, p. 2k-29).

3) A. Carmians, les caps harres de la regrion de Millau, Mémoires de la suciele des leellres de l'dueyrom, 19:21, tome : 1, p. $307-316$. 